\title{
Short and long-term outcomes after proximal gastrectomy with double tract reconstruction for Siewert type III adenocarcinoma of the esophagogastric junction: a propensity score matching study from a 10-year experience in a high-volume hospital
}

\author{
Fei Ma ${ }^{1 \#}$, Dandan Guo ${ }^{2 \#}$, Bin Zhang ${ }^{1}$, Yonglei Zhang ${ }^{1}$, Liangqun Peng ${ }^{1}$, Qi $\mathrm{Ma}^{1}$, Sheqing $\mathrm{Ji}^{1}$, Junhui Chai ${ }^{1}$, \\ Yawei Hua ${ }^{1}$, Xiaobing $\mathrm{Chen}^{3}$, Hui Wang ${ }^{3}$, Shuning $\mathrm{Xu}^{3}$, Suxia Luo ${ }^{3}$ \\ ${ }^{1}$ Department of General Surgery, the Affiliated Tumor Hospital of Zhengzhou University, Zhengzhou, China; ${ }^{2}$ Department of Radiology, The Third \\ Affiliated Hospital of Zhengzhou University, Zhengzhou, China; ${ }^{3}$ Department of Medical Oncology, the Affiliated Tumor Hospital of Zhengzhou \\ University, Zhengzhou, China \\ Contributions: (I) Conception and design: F Ma, S Luo; (II) Administrative support: Y Hua; (III) Provision of study materials or patients: B Zhang, \\ Y Zhang, Q Ma, S Ji, J Chai, X Chen; (IV) Collection and assembly of data: D Guo, L Peng, F Ma; (V) Data analysis and interpretation: D Guo, L \\ Peng, H Wang, S Xu; (VI) Manuscript writing: All authors; (VII) Final approval of manuscript: All authors. \\ \#These authors contributed equally to this work. \\ Correspondence to: Suxia Luo. Department of Medical Oncology, the Affiliated Tumor Hospital of Zhengzhou University, No. 127 Dongming Rd, \\ Zhengzhou 450008, China. Email: zlyyluosuxia0361@zzu.edu.cn.
}

Background: Total gastrectomy and proximal gastrectomy (PG) are both surgical options for the treatment of Siewert type III adenocarcinoma of the esophagogastric junction (AEG). Currently there is no consensus on selecting which procedure to perform; in particular, there are few reports of long-term outcomes for patients with local advanced AEG. The aim of this study was to validate the usefulness of PG with doubletract reconstruction in Siewert type III AEG.

Methods: The clinical data of patients with Siewert type III AEG underwent PG with double-tract reconstruction (PG-DT) or total gastrectomy with Roux-en-Y anastomosis (TG-RY) at our hospital between October 2010 and October 2018. According to the defined inclusion and exclusion criteria, 2,146 cases were enrolled in this study. A 1-to-1 propensity score matching (PSM) was performed to compare the short and long-term outcomes between the 2 groups.

Results: The operation time was longer in the PG-DT group, and the proportion rates of complications and recovery time was similar in the 2 groups. The rates of maintaining bodyweight and free-fat mass index were significantly higher in patients who underwent PG-DT compared to those who underwent TG-RY. While complications, recovery time and survival are similar between two groups.

Conclusions: Regarding short-term outcomes, PG-DT seemed to be superior in terms of maintaining body weight and skeletal muscle compared to TG-RY, while both had similar complications. It was found that PG-DT enabled a potentially longer survival of pathological stage II and III Siewert type III AEG, although the finding was statistically insignificant. These results may help surgeons to determine the appropriate surgical approach and strategy for patients with early and locally advanced Siewert type III AEG.

Keywords: Adenocarcinoma of the esophagogastric junction (AEG); proximal gastrectomy (PG); digestive tract reconstruction; double-tract reconstruction

Submitted Sep 27, 2020. Accepted for publication Dec 01, 2020.

doi: 10.21037/jgo-20-475

View this article at: http://dx.doi.org/10.21037/jgo-20-475

(c) Journal of Gastrointestinal Oncology. All rights reserved. 


\section{Introduction}

The incidence of adenocarcinoma of the esophagogastric junction is increasing $(1,2)$. Surgery-based comprehensive treatment is an effective treatment strategy for AEG, and is currently the only way to cure the disease (3). Total gastrectomy (TG) (4) and proximal gastrectomy (PG) (5) are the main surgical methods for Siewert types II and III AEG. However, while TG ensures an adequate resection margin and radical lymphadenectomy, it is also associated with poor nutritional status after surgery. Contrastingly, several studies have shown that PG can improve nutritional status and quality of life (QOL) compared with TG in early gastric cancer patients (6), perhaps because PG (5) preserves the physiological function of the gastric remnant. These benefits suggested that PG may be a better surgical approach for patients. A recent study indicated that there were no significant differences in the oncological safety of total gastrectomy and PG for local proximal gastric cancer (7). Therefore, PG has attracted broad interest from gastrointestinal oncologists in Eastern countries, including China.

However, PG with esophagogastrostomy has been associated with refractory reflux esophagitis and anastomotic stricture. These complications have limited the broader deployment of PG. Some other reconstruction methods have been explored to solve these complications, including side overlap esophagogastrostomy (8), doubleflap techniques (9), jejunal interposition $(10,11)$, double tract reconstruction $(12,13)$, and others. Due to comparable clinical benefits, $\mathrm{PG}$ with double-tract (DT) reconstruction may have become more prevalent in practice (14). A metaanalysis showed that PD with DT does not increase the risk of anastomotic stricture and reflux esophagitis as compared to TG (15). However, there are fewer reports on the survival of PG combined with DT.

This retrospective study was carried out to compare the long-term clinical outcomes of PG with DT (16) with those of TG with Roux-en-Y (RY) anastomosis (16) in patients with Siewert type III AEG. We present the following article in accordance with the STROBE reporting checklist (available at http://dx.doi.org/10.21037/jgo-20-475).

\section{Methods}

\section{Patient population}

The clinical data of patients with Siewert type III AEG who underwent proximal gastrectomy with double tract reconstruction (PG-DT) or TG-RY from October 2010 to October 2018 at The Affiliated Tumor Hospital of Zhengzhou University were included in this study. Patient selection was based on the following criteria: (I) Siewert type III AEG confirmed by gastroscopy and biopsy before surgery; (II) tumor size $<5 \mathrm{~cm}$; (III) abdominal enhanced computed tomography (CT) diagnosis was cT1-2N0M0; (IV) aged 18-80 years; (V) no history of malignant tumors and no serious underlying disease, Eastern Cooperative Oncology Group (ECOG) physical performance score $<2$, American Society of Anaesthesiologists (ASA) score $<3$ points; (VI) the patient provided written informed consent. Potential participants were excluded if the following criteria were met: (I) clinical stage > cT3 or $\mathrm{N}_{+}$; (II) patients who had not undergone radical surgery; (III) patient had undergone neoadjuvant therapy; (IV) patient had undergone a combined organ resection; (V) the patient had multiple malignant tumors; (VI) incomplete patient data.

Patients were divided into 2 groups according to surgical approach. The operation was determined by the operators' opinions. The participants were subjected to propensity score-matching analysis that was adjusted for several factors, namely, age, gender, ASA (17) physical status score (18), comorbidity, surgical approach, capillary invasion, nerve invasion, and pathological stage (Figure 1) to offset selection bias. The resulting matched TG and PG groups were compared in terms of demographic, clinical, surgical, and pathological characteristics, postoperative outcomes (including complications and nutrition status), and mortality during long-term follow-up.

The study was conducted in accordance with the Declaration of Helsinki (as revised in 2013). The study was approved by institutional ethics board of the affiliated tumor hospital of Zhengzhou University (No. 2019156) and informed consent was taken from all individual participants.

\section{Staging}

A CT scan of the chest and abdomen was performed on all participants. The clinical tumor stage was evaluated by 2 experienced roentgenologists according to the 8th edition of the International Union Against Cancer (UICC) staging system.

\section{Operative procedures}

All operations were performed via laparotomy or laparoscopy. The TG was performed according to the Japanese gastric cancer guidelines. 


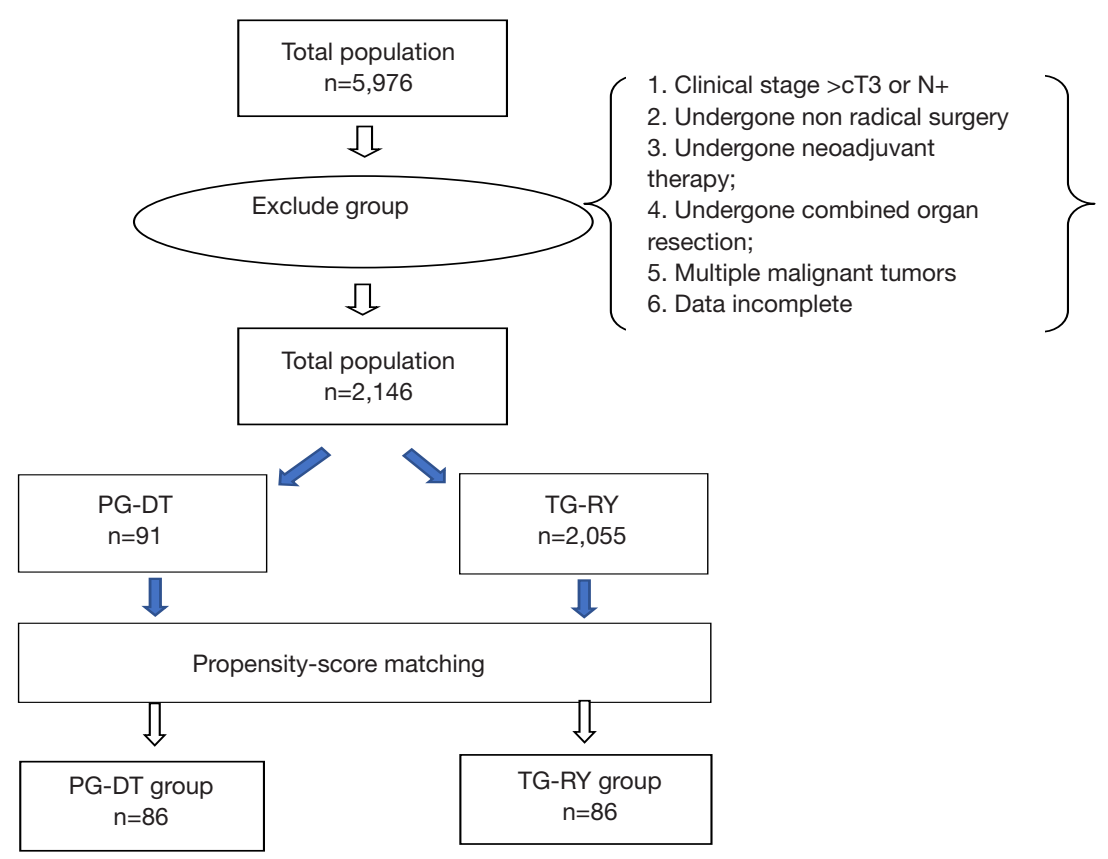

Figure 1 Flow chart of patient selection and propensity score matching. The 5,976 patients who underwent proximal gastrectomy with double-tract reconstruction (PG-DT) were matched to 2,146 patients who underwent total gastrectomy with Roux-en-Y anastomosis (TG-RY) in terms of age, gender, American Society of Anaesthesiologists (ASA) physical status score (17), comorbidity, surgical approach, capillary invasion, nerve invasion, and pathological tumor, node, metastasis (TNM) stage.

For PG, the lymph node dissection was performed with extended D1+ lymphadenectomy including dissection of the lymph nodes at station no. 1, 2, 3, 4sa, 4sb, 5, 7, 8a, 9, 11p, 12a, and incomplete no. $4 \mathrm{~d}$ (outside of greater curvature), and no. 6 [anterosuperior lymph nodes (LNs) along gastroepiploic vessels]. The anastomosis was performed using the DT reconstruction method, and the details were as follows: the patient was placed in a supine position under general anesthesia, and a midline incision was made in the upper abdomen. After a thorough examination to exclude abdominal metastasis, the PG was performed. The omentum was separated to the edge of nos. $4 \mathrm{~d}$ and 6 lymph nodes, and right gastroepiploic artery and vein were preserved. The no. 12a lymph nodes were dissected, right gastric artery and vagal nerves were cut off, and the no. 5 and no. $3 \mathrm{~b}$ lymph nodes were dissected. The small omentum was dissected to the right crus of the diaphragmatic. The stomach was divided with a linear stapler while ensuring the distal resection margin. Station no. 7, 8a, 9, and 11p lymph nodes were then dissected, the left gastric artery, coronary vein, and posterior gastric vessels were ligated next. The left gastroepiploic vessels were ligated, and the station no. 4 sb lymph nodes were dissected. The short gastric vessels were ligated, and the no. 10 and no. 4sa lymph nodes were dissected and expanded to the left crus of the diaphragmatic.

The reconstruction methods were divided into a laparotomy anastomosis group and a laparoscopic anastomosis group. In the conventional anastomosis group, the anvil of the circular stapler was placed into the stump of the esophagus and the stomach remnant separately, using a purse-string suturing device. The jejunum was divided about $25 \mathrm{~cm}$ below the ligament of Treitz, and a circular stapler was inserted into the distal jejunal opening. Gastrojejunostomy was performed about $15 \mathrm{~cm}$ below the distal jejunal opening on the posterior wall of the remnant stomach, and then esophagojejunostomy was performed, after which, the linear stapler was used to close the jejunal opening. Jejunojejunostomy was performed about $45 \mathrm{~cm}$ below the esophagojejunostomy. All anastomoses were intermittent or continuous sutured again for reinforcement (Figure 2). A silicone drainage tube was inserted at the posterior parts of esophagojejunostomy and led out from the right abdominal wall through the hepatoduodenal ligament behind. In the laparoscopic anastomosis group, each anastomosis site was marked on the alimentary limb similar to the conventional approach, where esophagojejunostomy 

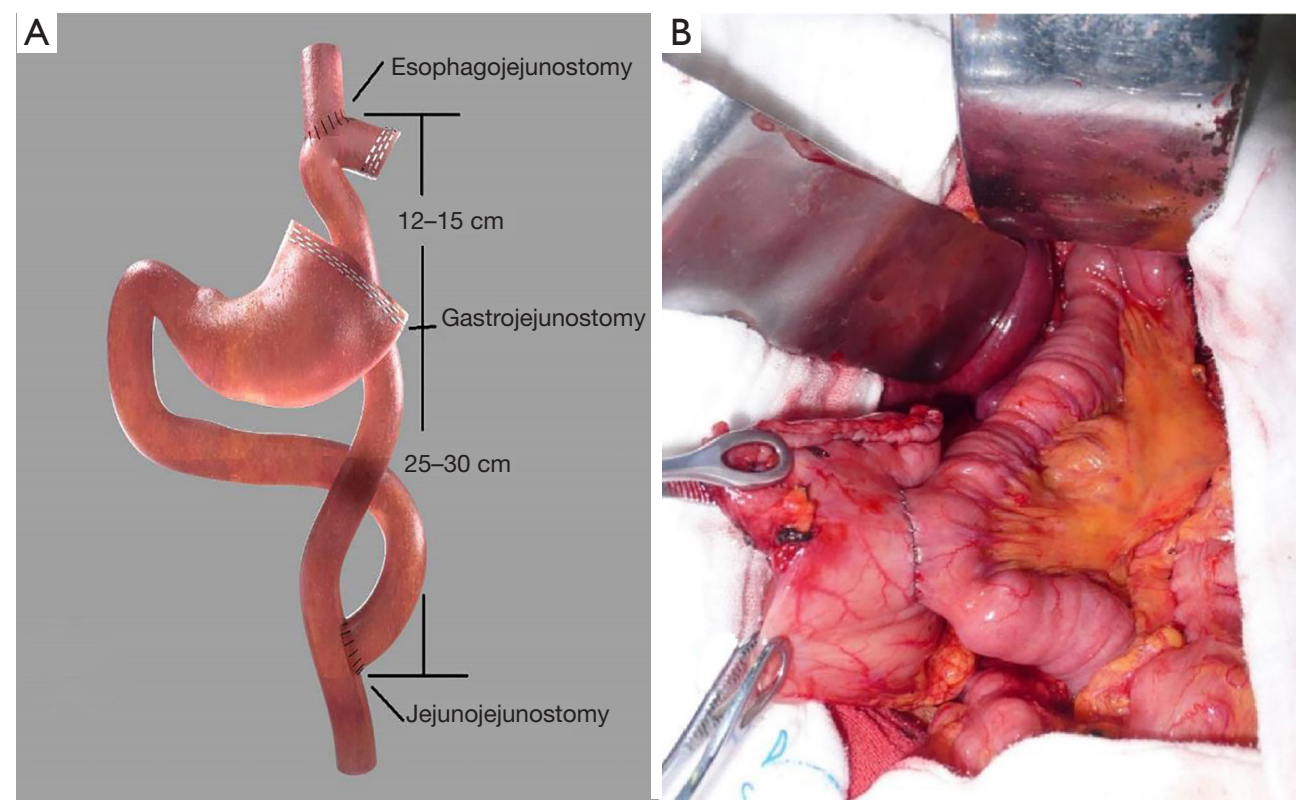

Figure 2 Figuration of proximal gastrectomy with double tract reconstruction. (A) Schematic diagram of proximal gastrectomy with double tract reconstruction; (B) anastomosis in the laparotomy group.

and gastrojejunostomy were performed using a laparoscopic linear stapler. Hand-sewn jejunojejunostomy was performed extracorporeally via mini-laparotomy (Figure 3).

\section{Postoperative therapy and follow-up}

Postoperative adjuvant chemotherapy with platinum and fluorouracil was principally administered based on the National Comprehensive Cancer Network (NCCN) guidelines from 2008 (19). Overall survival (OS) was defined as the period from the initiation of surgery to any cause of death and recurrence-free survival (RFS) as a period from the initiation of surgery to recurrence or death. All patients were outpatient followed up every 3-6 months. Physical examination and hematologic analysis were performed at each visit, including tumor marker evaluation for carcinoembryonic antigen (CEA), carbohydrate antigen 72-4 (CA72-4), and carbohydrate antigen 19-9 (CA19-9). Bodyweight and Fat-Free Mass Index (FFMI) were measured using InBody570 (Biospace, Co., Ltd., Urbandale, IA, USA). Chest and abdominal CT was performed every 6 months or when recurrence was clinically suspected. Gastroscopy was performed every 12 months, and these endoscopic findings were scored in terms of the Los Angeles classification of reflux esophagitis (20). The survival status of the patients was determined in June 2020, and the median follow-up was 48 [26-72] months. A total of 6 participants (3.9\%) were lost at follow-up.

\section{Statistical analyses}

Propensity score matching was based on group and involved 1-to-1 pair matching without replacement using a caliper of 0.05.well matched. No covariate exhibited a large imbalance $(|\mathrm{d}|>0.25)$. The continuous variables were all normally distributed and are therefore expressed as mean and standard deviations, while categorical variables are expressed as n (\%). The TG and PG groups were compared in terms of continuous variables using Student's $t$-test and in terms of categorical variables using the chi-squared $\left(\chi^{2}\right)$ test or Fisher's exact test. The OS was calculated from the date of surgery until the final follow-up or death from any cause. Survival curves were generated using the KaplanMeier method. The groups were compared in terms of survival using the log-rank test. All analyses were conducted using SPSS for Windows version 21.0 (SPSS, Inc., IBM, Chicago, IL, USA). The $\mathrm{P}$ values of $<0.05$ were considered to indicate statistically significant differences.

\section{Results}

The 5,976 patients who underwent PG-DT were matched 

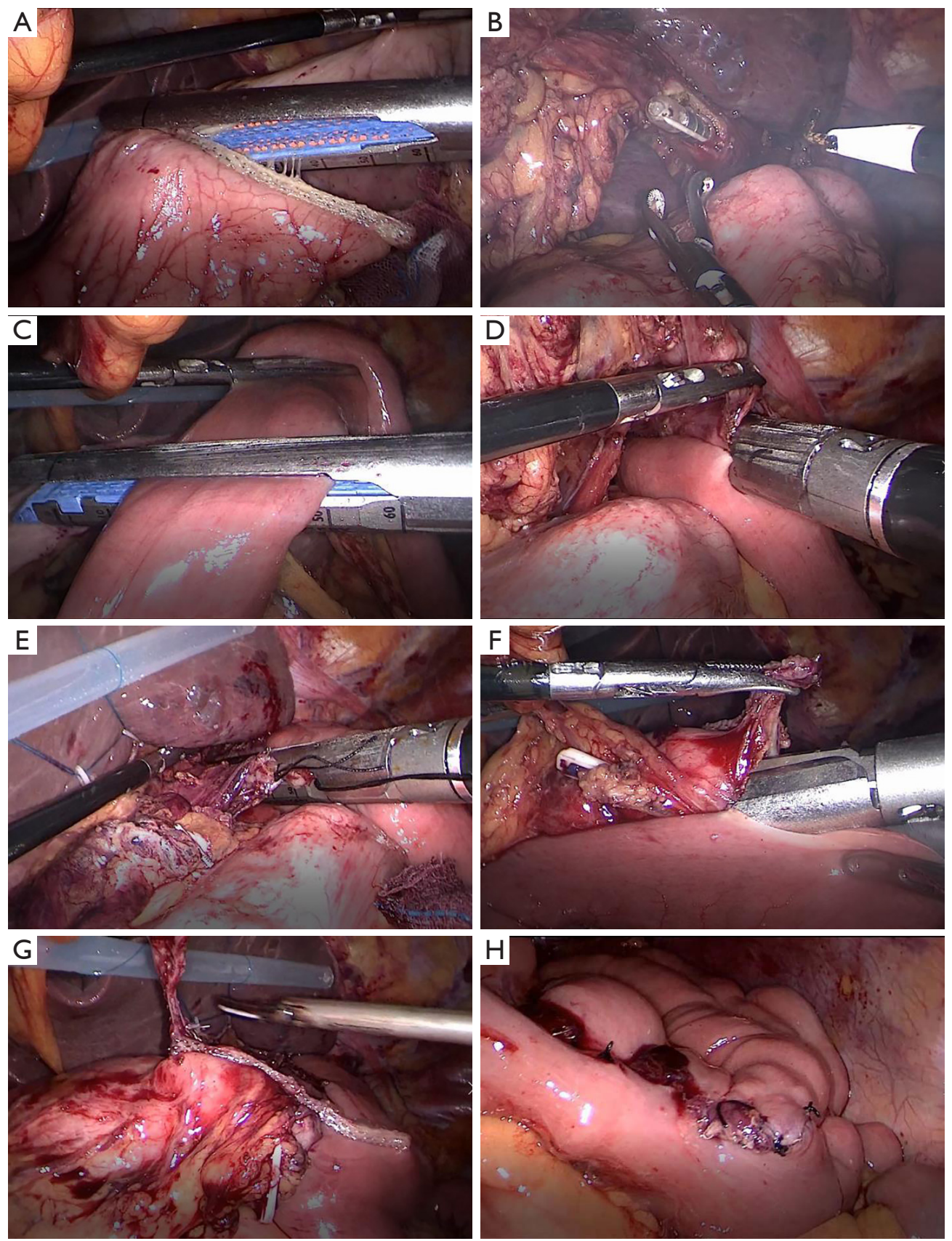

Figure 3 Laparoscopic surgery procedure. (A) Divided stomach; (B) gastric tube guidance of the esophagus; (C) divided jejunum about $25 \mathrm{~cm}$ below the ligament of Treitz; (D) over-lap esophagojejunostomy; (E) laparoscopic linear stapler closes common opening; (F) side-toside gastrojejunostomy; $(\mathrm{G})$ laparoscopic linear stapler closes common opening; (H) hand-sewn jejunojejunostomy.

to 2,146 patients who underwent TG-RY in terms of age, sex, ASA (17) physical status score, comorbidity, surgical approach, capillary invasion, nerve invasion, and pathological TNM stage.

A total of 2,146 cases were included in this study, 2,055 cases underwent total gastrectomy, 91 cases underwent radical PG with double tract reconstruction. Before PSM, there was no statistically significant difference between the two groups in terms of age, sex, comorbidity, ASA, surgical approach, capillary invasion and nerve invasion. However, 
TNM stage of the TG-RY group were later than the PGDT group and the differences were statistically significant. After PSM, the two groups based on the above eight factors, a total of 172 patients were included in the study, and the differences were evenly distributed between the two groups. The clinicopathological characteristics of the patients are shown in Table 1.

The operation time in TG-RY group and PG-DT group were $(164 \pm 17)$ and $(177 \pm 30) \mathrm{min}$, respectively, and the difference was statistically significant $(\mathrm{P}<0.05)$. The blood loss in two groups were $(106 \pm 62)$ and $(91 \pm 32) \mathrm{mL}$, the number of lymph node dissections in two groups were $25 \pm 7$ and $23 \pm 6$, respectively, all of those differences were not statistically significant $(\mathrm{P}>0.05)$. The recovery time of gastrointestinal function in two groups were $(3.3 \pm 0.9)$ days and $(2.2 \pm 0.9)$ days, the feeding time of two groups were $(5.9 \pm 3.7)$ and $(5.6 \pm 2.7)$ days, the postoperative hospital stay were $(10.5 \pm 2.1)$ and $(11.3 \pm 3.4)$ days respectively, the difference was not statistically significant $(\mathrm{P}>0.05)$. Three cases of severe complications were occurred in TG-RY group included 1 cases of anastomotic leakage (IIIa), 1 case of anastomotic bleeding (IIIa), 1 cases of Anastomotic stenosis (IIIa). And 2 cases of severe complications were occurred in PG-DT group included anastomotic leakage (IIIa). There was no statistically significant difference between the two groups in terms of the incidence of severe complications and it's classifications $(\mathrm{P}>0.05)$. The shortterm outcomes are shown in Table 2.

The patients in the PG-DT group exhibited significantly lower weight loss and FFMI loss than those in the TG group $(\mathrm{P}<0.05)$ (Figure 4).

The 5-year OS rates for patients with pathological stage I in PG-DT and TG-RY were 90.7\% [95\% confidence interval (CI): $78.2-100 \%]$ and $91.4 \%$ (95\% CI: 79.4-100\%), respectively $(\mathrm{P}=0.904)$. The 5 -year RFS rates for PG-DT and TG-RY were $90.8 \%$ (95\% CI: $78.5-100 \%$ ) and $95.5 \%$ (95\% CI: 86.9-100\%), respectively ( $\mathrm{P}=0.490)$.

The 5-year OS rates for patients with pathological stage II in PG-DT and TG-RY were $71.5 \%$ (95\% CI: $53.5-$ $89.5 \%$ ) and $65.3 \%$ (95\% CI: 43.9-86.7\%), respectively $(\mathrm{P}=0.365)$. The 5 -year RFS rates for PG-DT and TG-RY were $64.4 \%$ (95\% CI: $43.8-85.0 \%$ ) and $60.4 \%$ (95\% CI: $38.4-82.4 \%)$, respectively $(\mathrm{P}=0.490)$.

The 5-year OS rates for patients with pathological stage III in PG-DT and TG-RY were 51.9\% (95\% CI: $31.7-$ $72.1 \%$ ) and $42.1 \%$ (95\% CI: 22.3-61.9\%), respectively $(\mathrm{P}=0.272)$. The 5 -year RFS rates for PG-DT and TG-RY were $44.7 \%$ (95\% CI: 24.9-64.5\%) and 32.9\% (95\% CI:
14.1-51.7\%), respectively ( $\mathrm{P}=0.354)$ (Figure 5).

\section{Discussion}

The present study was performed to clarify the merits of PG-DT over TG-RY in patients with clinical stages T1-2N0M0, explicitly focusing on the survival based on pathological staging as well as surgical complications and body composition changes. The proportion of complications for patients who underwent PG-DT was low in our study, and the postoperative recovery was favorable. There was no difference between PG-DT and TG-RY in terms of the incidence of complications and classifications. Moreover, PG has delivered an improvement in nutritional status, especially regarding the mass of skeletal muscle. Most importantly, although there is no statistical difference, compared with TG, PG seems to improve the survival of local advanced Siewert type III AEG.

The scope of surgical resection and reconstruction of AEG is still controversial despite various attempts at clarification. Relative to TG, multiple studies have reported the non-inferiority of PG for early AEG and upper gastric body cancer $(4,20,21)$. The anastomosis of the esophagus after PG is the simplest method, but it leads to obvious reflux esophagitis, reflux symptoms, and anastomotic stenosis $(20,22)$. However, there are few reports on the long-term survival of patients with AEG after PG-DT. Technical complexity is a concern for most surgeons due to the increased number of anastomoses. Postoperative complications did increase in previous patients with jejunal interposition despite the excellent anti-reflux effects, such as intestinal obstruction, anastomosis stricture, and food residue (23-25). However, these were improved in DT (5). At the beginning of our laparoscopic PG-DT, operation time was as long as 470 minutes. After optimizing the process, the operation time was shortened rapidly. This situation was similar to the findings of Uyama et al. (26).

The current recommendations on the extent of lymphadenectomy for proximal gastric AEG have always been controversial. The probability of lymph node metastasis in early gastric cancer (EGC) is much lower than that in advanced forms. However, the risk still ranges considerably according to pathological characteristics. In EGC unsuitable for endoscopic resection, the Japanese Gastric Cancer Association (JGCA) guidelines advised a D1 or D1 plus lymphadenectomy when lymph nodes are clinically negative, and when lymph nodes are clinically positive, D2 dissection should be performed. Unfortunately, 
Table 1 Patient characteristics before and after PSM

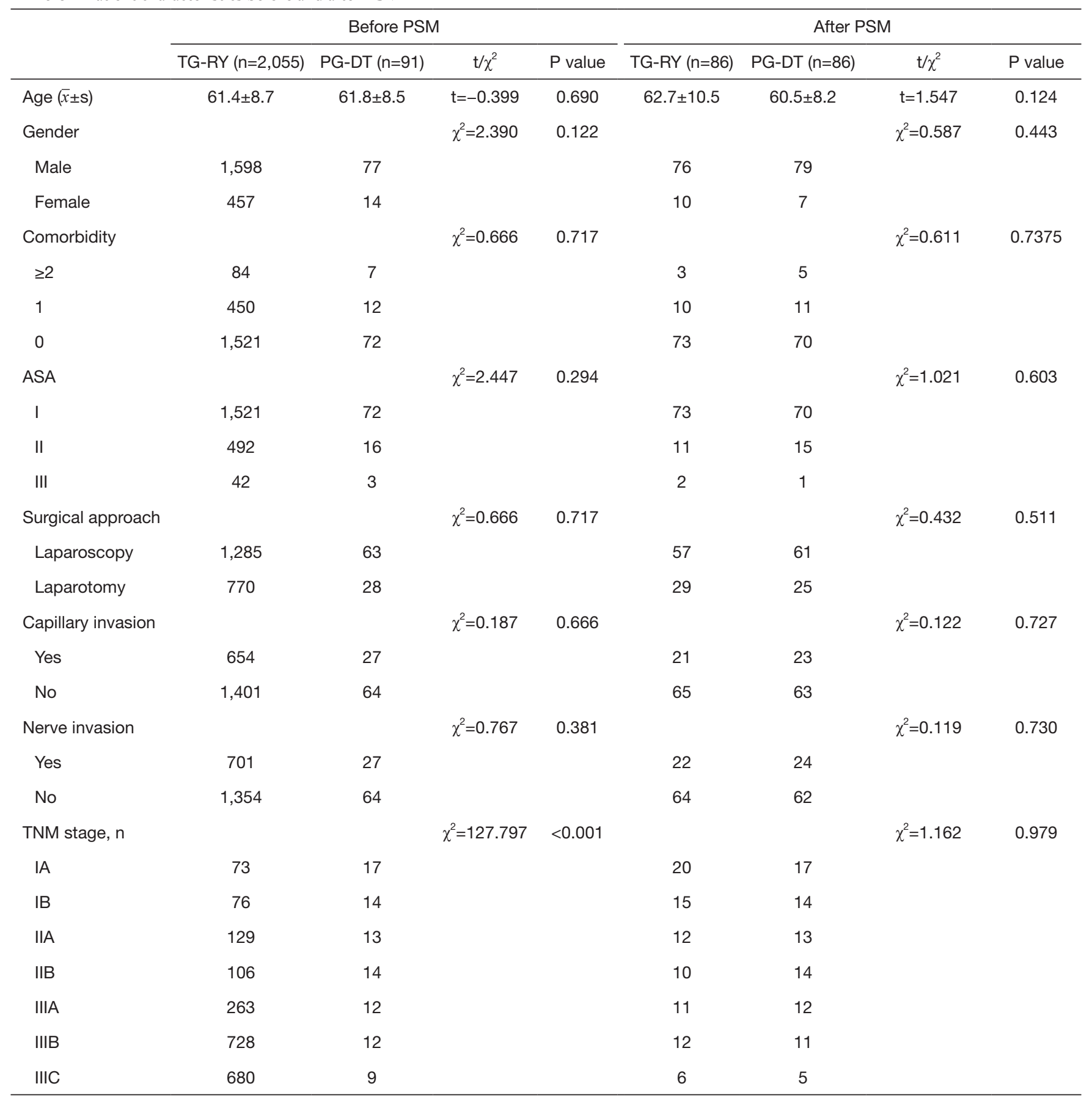

PSM, propensity score matching; TG-RY, total gastrectomy with Roux-en-Y anastomosis; PG-DT, proximal gastrectomy with double-tract reconstruction; ASA, American Society of Anaesthesiologists; TNM, tumor, node, metastasis. 
Table 2 Short-term outcomes after PSM

\begin{tabular}{|c|c|c|c|c|}
\hline & TG-RY $(n=86)$ & PG-DT (n=86) & $t / \chi^{2}$ & $P$ value \\
\hline Blood loss (mL) & $106 \pm 62$ & $91 \pm 32$ & $t=1.878$ & 0.062 \\
\hline Number of lymph nodes obtained (piese) & $25 \pm 7$ & $23 \pm 6$ & $t=1.435$ & 0.153 \\
\hline Exhaust time (day) & $3.3 \pm 0.9$ & $2.2 \pm 0.9$ & $t=0.443$ & 0.658 \\
\hline Postoperative hospital stay (day) & $10.5 \pm 2.1$ & $11.3 \pm 3.4$ & $t=1.819$ & 0.071 \\
\hline Complications classification & & & $\chi^{2}=0.124$ & 0.724 \\
\hline $\mathrm{I} / \mathrm{II}$ & 4 & 4 & & \\
\hline III/IV & 3 & 2 & & \\
\hline Anastomotic bleeding & 1 & 0 & & \\
\hline Anastomotic stenosis & 1 & 0 & & \\
\hline Intestinal obstruction & 0 & 1 & & \\
\hline Lung infection & 1 & 2 & & \\
\hline Chyle leak & 1 & 1 & & \\
\hline Incision infection & 2 & 0 & & \\
\hline
\end{tabular}

PSM, propensity score matching; TG-RY, total gastrectomy with Roux-en-Y anastomosis; PG-DT, proximal gastrectomy with double-tract reconstruction.

the definitions of the D2 level of lymph node dissection were only for TG and distal gastrectomy; PG was not included (27). Local advanced gastric cancer had a high tendency to lymph nodes involvement and spread. Lymph node dissection has been debated for several years by surgeons between Eastern and Western countries. The D2 lymphadenectomy has been considered the standard procedure in Japan since the 60's, but the opinions of surgeons in Western countries differ, according to Western randomized clinical trials (28). It is unfortunate that some relatively early proximal gastric cancer patients have undergone TG, which has meant a decreased QOL for them. Prior to 2010, there were few uniform clinical research results on the scope of surgical lymph node dissection for local advanced upper gastric cancer or AEG. At the time, it was difficult to ascertain whether to perform lymph node dissection of the proximal stomach. We also found that there was a discrepancy between preoperative imaging staging and postoperative pathology due to some objective reasons. Therefore, referring to the Japanese
Gastric Cancer Guidelines for that time (29), we developed a range of lymph node dissection options for PG, which was similar to the D2 lymphadenectomy for TG, including dissection of the lymph nodes at stations no. 1, 2, 3, 4sa, 4sb, 5, 7, 8a, 9, 11p, 12a, incomplete no. 4d (outside of greater curvature), and no. 6 (anterosuperior LNS along gastroepiploic vessels), or it can be called incomplete D2 lymphadenectomy for PG. We observed that as such incomplete D2 lymphadenectomy did not increase the patient's surgical risk; additionally, in our previous study, we found that PG-DT could improve patient nutritional status and QOL (30). Not all researchers have come to similar conclusions; Yamashita et al. reported the incidences of metastasis at node nos. $4 \mathrm{sa}, 4 \mathrm{sb}, 4 \mathrm{~d}, 5$, and 6 were $<1 \%$, even in patients with high dissection rates in stomachpredominant cancer from results of their nation-wide retrospective study for AEG in Japan (31). However, results from the China National Cancer Center Gastric Cancer Database during 1997-2017 revealed that proximal gastric cancer patients were more likely to be in later $\mathrm{pT}$ stage 

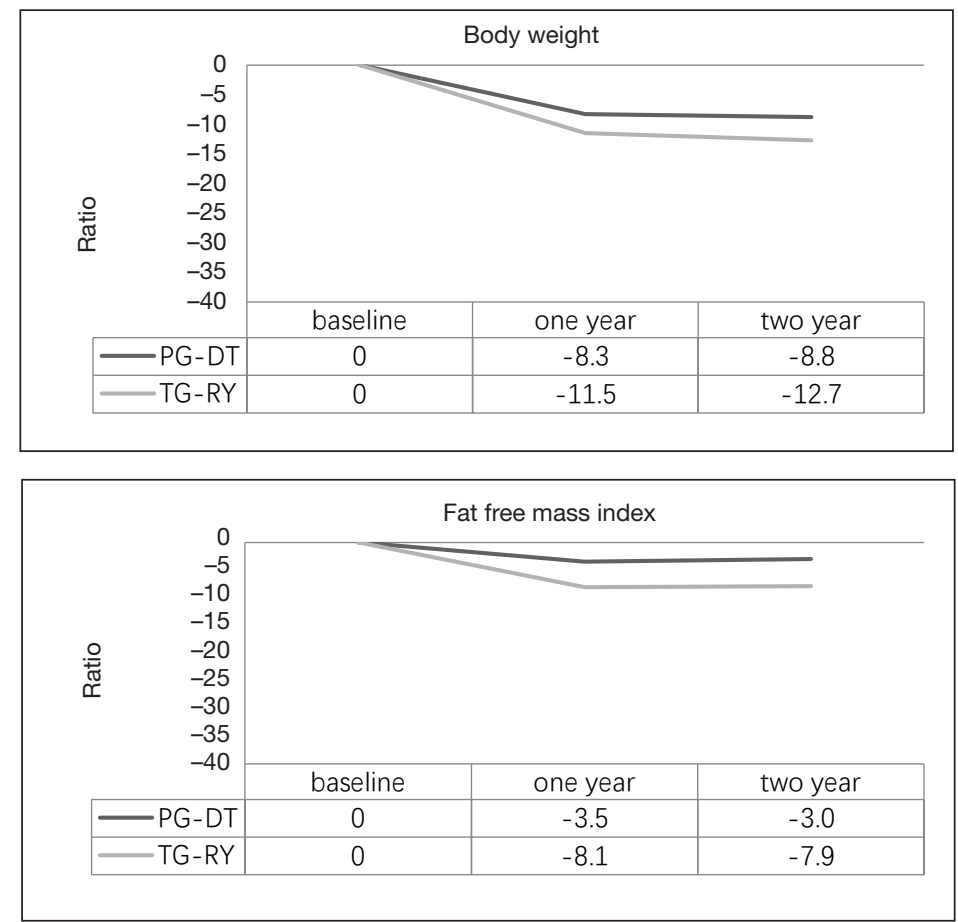

Figure 4 Bodyweight and FFMI. The patients in the PG group exhibited significantly lower weight loss and FFMI loss than those in the TG group $(\mathrm{P}<0.05)$. FFMI, fat-free mass index; $\mathrm{PG}$, proximal gastrectomy; TG, total gastrectomy.

and lymph node metastasis; Wang et al. reported the rates of metastasis in the lymph node groups in advanced PGC ranged from high to low in the lymph node no. 3, 1, 2, 7, $4,8 \mathrm{a}, 10,11,6$, and. 5 groups. For patients at pT1-3, the positive rate for lymph nodes no. 5 and 6 was $15.1 \%$ (32). Considering the discrepancy between preoperative clinical staging and postoperative pathological staging, use of this type of lymph node dissection has always been controversial.

There seems to be a consensus on the nutritional benefits of PG compared to TG. The PG is more beneficial than TG in serum albumin levels, maintaining weight, in terms of preventing anemia, and maintaining serum vitamin B12 levels. However, the late stenosis and reflux symptoms caused by anastomosis of the esophagus and remnant stomach must be addressed (33). Jung et al. reported that PG with jejunal interchannel reconstruction did not increase the incidence of complications such as reflux, and had significant advantages over TG in terms of weight, anemia, and serum vitamin B12 level (34). We reached a similar conclusion regarding serum albumin values and the maintenance rates of body mass index (BMI) in our previous study (35). A Japanese nation-wide multiinstitutional surveillance study between patients who had undergone PG and TG revealed that bodyweight loss was significantly lower with PG (36), and these findings were consistent with Asaoka et al. (37). In the present study, we found that patients with PG have less FFMI reduction than TG, Consistent with our findings, Sugiyama et al. showed the skeletal muscle index reduction rate of the LPG-DT group in the first postoperative year was significantly lower than that of the LTG group (38). Although the detailed mechanism of body weight and skeletal muscle maintenance is not explained in this study, there are some possible explanations. The food intake of PG patients may be more than that of TG patients; the secretion of the known or unknown gut hormones may help to increase the appetite or food reservoir; the absorptive function may be better due to the bile acid and pancreatic juice being well-mixed with food as it passes through the duodenum (39). The impact of the relationship between the motor activity of the remnant gastric, duodenum, and jejunal pouch must also be considered (40).

For PG, the safety of oncology is the first consideration. An et al. reported that 5 -year survival rates were similar after TG and PG for patients with early gastric cancer in the upper third of the stomach (21). Laparoscopy- 

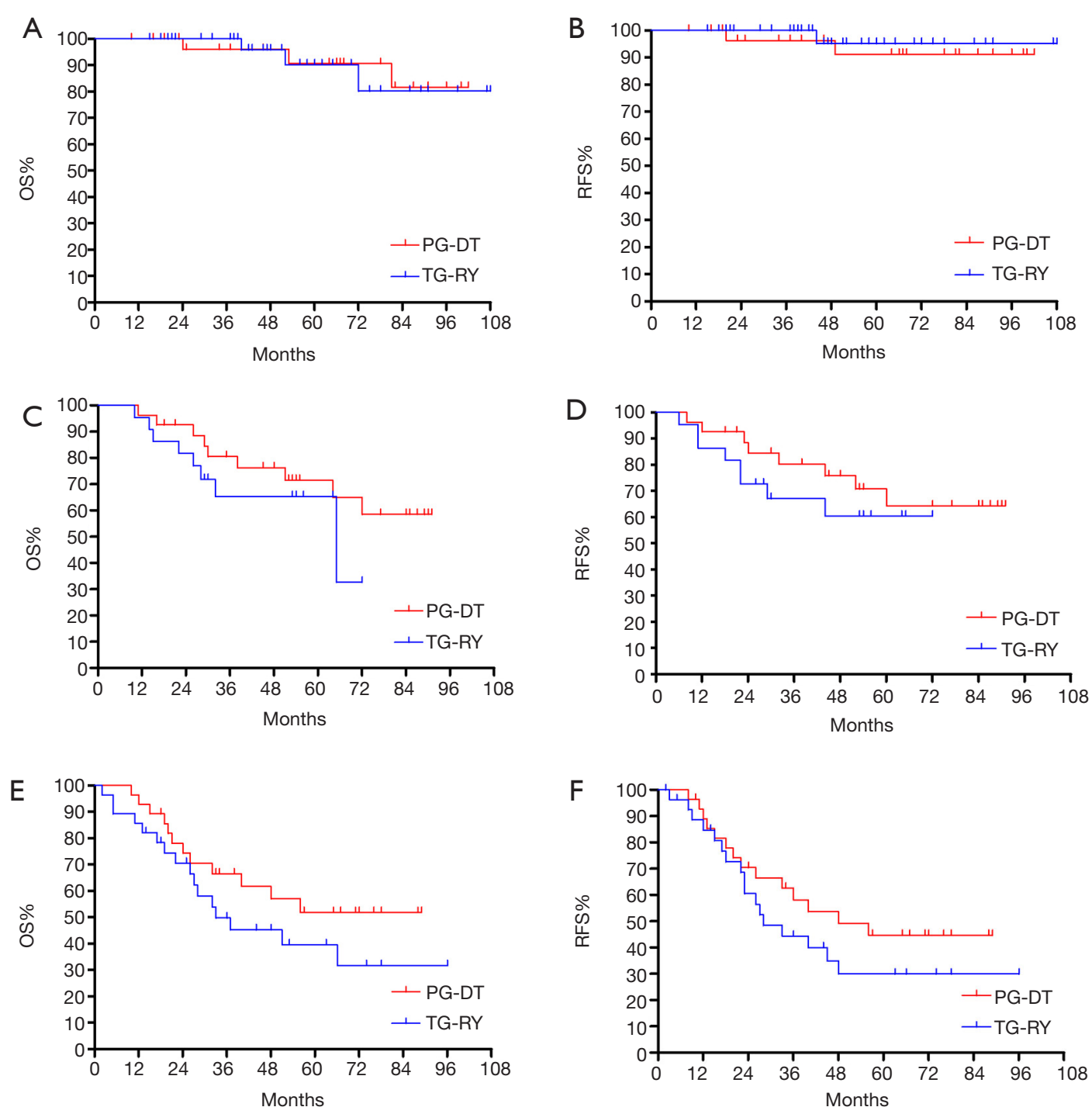

Figure 5 Kaplan-Meier survival curves for OS (A) and RFS (B) in the pathological stage I population. Kaplan-Meier curves for OS (C) and RFS (D) in pathological stage II patients. Kaplan-Meier curves for OS (E) and RFS (F) in pathological stage III patients. OS, overall survival; RFS, recurrence-free survival.

assisted TG (LATG) or laparoscopy-assisted PG (LAPG) is expected to be established as one of the standard treatments for clinical stage I gastric cancer in the JCOG1401 trial (41). We reached the same conclusion in EGC. However, it remained that although there was no statistical difference, compared with TG, in the case of sufficient lymph node dissection, PG-DT appears to tend to improve the survival of local advanced Siewert type III AEG. Although the detailed mechanism of survival improvements is not clear yet, we may hypothesize some possible explanations. First, for local advanced Siewert type
III AEG, PG and similar to the D2 lymphadenectomy for TG was sufficient and appropriate (incomplete no. $4 \mathrm{~d}$ and no. 6), Although precise $T$ staging has a clear guiding effect on surgical methods (42), there is still the possibility of objective inconsistency between preoperative clinical staging and postoperative pathological staging. Wang et al. reported that in the T1-T3 stage or the tumor size $<5 \mathrm{~cm}$, there was no significant difference between the 5-year survival rate of patients with and without metastasis in lymph nodes no. 5, 6 (48.7\% vs. 49.3\%, $\mathrm{P}=0.755$ ) (32). The data obtained from 2 high-volume institutions in South China showed that 
with the routinely performed sufficient lymphadenectomy, the survival of patients gradually improved from 1991 to 2010 (43). Second, the improvement of nutritional status may have played a significant role. Our present study showed that PG-DTR could reduce reflux esophagitis while significantly improving the nutritional status of patients and reducing the appearance of sarcopenia. Although the proportion of food passing through the remnant stomach cannot be accurately determined, it was still surprisingly found (through retrospective analysis) that the reduction in FFMI of PG-DTR was significantly better than that of TG-RY (44). Sarcopenia refers to skeletal muscle loss with aging or for other reasons. The development of sarcopenia as a result of a decrease in skeletal muscle might worsen postoperative survival (45). Performance of PG-DT might be considered to offer a good ability to maintain QOL after surgery. The prognosis of EGC is generally good, and more prolonged observation of whether there is a loss of survival in EGC, is required. For stage III gastric cancer, the tumor progresses rapidly, and the role of nutrition seems to be weakened. Third, with the improvement of nutritional status and QOL, the chemotherapy tolerance of PG may be better than TG. Moreover, the difference in trauma caused by surgery seems to be another possible factor. However, some doctors may have concerns about choosing PG due to the risk of occurrence of residual gastric cancer, of which survival is relatively poor (46).

There were several limitations to this study. First, this is a retrospective study that lasted for 10 years. Even though we have used propensity score analysis, we still cannot altogether avoid a certain degree of selection bias. The surgical procedure in each case was selected based on the collaborative decision between the surgeon and patient, and the location of the tumor. Second, the follow-up time was short, and the ongoing oncology results could not be accurately assessed.

\section{Conclusions}

At present, there is no consensus on the surgical method for Siewert type III AEG. We analyzed and compared the clinical outcomes of PG with DT and TG with RY for Siewert type III AEG over the past 10 years, and found the former to be superior in terms of maintaining body weight and skeletal muscle. In particular, it was found that PGDT offered a longer survival of pathological stage II and III Siewert type III AEG through subgroup analysis, although this finding was statistically insignificant. These results may help surgeons to determine an appropriate surgical approach and strategy for patients with early and local advanced Siewert type III AEG.

\section{Acknowledgments}

Funding: The study was supported by Tackle Key Problems of Henan Province (202102310414), Tackle Key Problems in Medicine of Henan Province (LHGJ20190629), and the National Cancer Climbing Fund (no. NCC201816B048). The funding sources had no role in study design, data collection, data analysis, data interpretation, or writing of the report.

\section{Footnote}

Reporting Checklist: The authors have completed the STROBE reporting checklist. Available at http://dx.doi. org/10.21037/jgo-20-475

Data Sharing Statement: Available at http://dx.doi. org/10.21037/jgo-20-475

Conflicts of Interest: All authors have completed the ICMJE uniform disclosure form (available at http://dx.doi. org/10.21037/jgo-20-475). The authors have no conflicts of interest to declare.

Ethical Statement: The authors are accountable for all aspects of the work in ensuring that questions related to the accuracy or integrity of any part of the work are appropriately investigated and resolved. The study was conducted in accordance with the Declaration of Helsinki (as revised in 2013). The study was approved by institutional ethics board of the affiliated tumor hospital of Zhengzhou University (No. 2019156) and informed consent was taken from all individual participants.

Open Access Statement: This is an Open Access article distributed in accordance with the Creative Commons Attribution-NonCommercial-NoDerivs 4.0 International License (CC BY-NC-ND 4.0), which permits the noncommercial replication and distribution of the article with the strict proviso that no changes or edits are made and the original work is properly cited (including links to both the formal publication through the relevant DOI and the license). See: https://creativecommons.org/licenses/by-nc$\mathrm{nd} / 4.0 /$. 


\section{References}

1. Bray F, Ferlay J, Soerjomataram I, et al. Global cancer statistics 2018: GLOBOCAN estimates of incidence and mortality worldwide for 36 cancers in 185 countries. CA Cancer J Clin 2018;68:394-424.

2. Perkhofer L, Hann A. Single nucleotide polymorphisms as the new predictors of therapy decisions in gastroesophageal junction and gastric adenocarcinoma? Transl Cancer Res 2019;8:1656-8.

3. Greally M, Agarwal R, Ilson DH. Optimal management of gastroesophageal junction cancer. Cancer 2019;125:1990-2001.

4. Ahn SH, Lee JH, Park DJ, et al. Comparative study of clinical outcomes between laparoscopy-assisted proximal gastrectomy (LAPG) and laparoscopy-assisted total gastrectomy (LATG) for proximal gastric cancer. Gastric Cancer 2013;16:282-9.

5. Ahn SH, Jung DH, Son SY, et al. Laparoscopic doubletract proximal gastrectomy for proximal early gastric cancer. Gastric Cancer 2014;17:562-70.

6. Xu Y, Tan Y, Wang Y, et al. Proximal versus total gastrectomy for proximal early gastric cancer: A systematic review and meta-analysis. Medicine (Baltimore) 2019;98:e15663.

7. Yura M, Yoshikawa T, Otsuki S, et al. Oncological safety of proximal gastrectomy for T2/T3 proximal gastric cancer. Gastric Cancer 2019;22:1029-35.

8. Yamashita Y, Yamamoto A, Tamamori Y, et al. Side overlap esophagogastrostomy to prevent reflux after proximal gastrectomy. Gastric Cancer 2017;20:728-35.

9. Kuroda S, Nishizaki M, Kikuchi S, et al. DoubleFlap Technique as an Anti-reflux Procedure in Esophagogastrostomy after Proximal Gastrectomy. J Am Coll Surg 2016;223:e7-e13.

10. Kameyama J, Ishida H, Yasaku Y, et al. Proximal gastrectomy reconstructed by interposition of a jejunal pouch. Surgical technique. Eur J Surg 1993;159:491-3.

11. Nomura E, Lee SW, Kawai M, et al. Functional outcomes by reconstruction technique following laparoscopic proximal gastrectomy for gastric cancer: double tract versus jejunal interposition. World J Surg Oncol 2014;12:20.

12. De Ferrari GM, Dusi V, Spazzolini C, et al. Response to Letters Regarding Article, "Clinical Management of Catecholaminergic Polymorphic Ventricular Tachycardia: The Role of Left Cardiac Sympathetic Denervation". Circulation 2016;133:e366-7.

13. Aikou T, Natsugoe S, Shimazu H, et al. Antrum preserving double tract method for reconstruction following proximal gastrectomy. Jpn J Surg 1988;18:114-5.

14. Wang S, Lin S, Wang H, et al. Reconstruction methods after radical proximal gastrectomy: A systematic review. Medicine (Baltimore) 2018;97:e0121.

15. Li S, Gu L, Shen Z, et al. A meta-analysis of comparison of proximal gastrectomy with double-tract reconstruction and total gastrectomy for proximal early gastric cancer. BMC Surg 2019;19:117.

16. Fan JY, Qian F, Liu JJ, et al. Comparison of clinical efficacy between proximal gastrectomy with double tract reconstruction and total gastrectomy with Roux-en-Y reconstruction for proximal gastric cancer. Zhonghua Wei Chang Wai Ke Za Zhi 2019;22:767-73.

17. Tanaka K, Ebihara Y, Kurashima Y, et al. Laparoscopic proximal gastrectomy with oblique jejunogastrostomy. Langenbecks Arch Surg 2017;402:995-1002.

18. A P, Bd A, Wm Z, et al. Knowledge, Attitudes, Practices and Health Beliefs toward Leptospirosis among Urban and Rural Communities in Northeastern Malaysia. Int J Environ Res Public Health 2018;15:2425.

19. Kubo M, Sasako M, Gotoda T, et al. Endoscopic evaluation of the remnant stomach after gastrectomy: proposal for a new classification. Gastric Cancer 2002;5:83-9.

20. Masuzawa T, Takiguchi S, Hirao M, et al. Comparison of perioperative and long-term outcomes of total and proximal gastrectomy for early gastric cancer: a multi-institutional retrospective study. World J Surg 2014;38:1100-6.

21. An JY, Youn HG, Choi MG, et al. The difficult choice between total and proximal gastrectomy in proximal early gastric cancer. Am J Surg 2008;196:587-91.

22. Huh YJ, Lee HJ, Oh SY, et al. Clinical Outcome of Modified Laparoscopy-Assisted Proximal Gastrectomy Compared to Conventional Proximal Gastrectomy or Total Gastrectomy for Upper-Third Early Gastric Cancer with Special References to Postoperative Reflux Esophagitis. J Gastric Cancer 2015;15:191-200.

23. Seshimo A, Miyake K, Amano K, et al. Clinical outcome of esophagogastrostomy after proximal gastrectomy for gastric cancer. Hepatogastroenterology 2013;60:616-9.

24. Katai H, Morita S, Saka M, et al. Long-term outcome after proximal gastrectomy with jejunal interposition for suspected early cancer in the upper third of the stomach. Br J Surg 2010;97:558-62.

25. Nakamura $M$, Nakamori $M$, Ojima T, et al. Reconstruction after proximal gastrectomy for early gastric cancer in the upper third of the stomach: an analysis of our 13-year experience. Surgery 2014;156:57-63. 
26. Uyama I, Sugioka A, Fujita J, et al. Completely laparoscopic proximal gastrectomy with jejunal interposition and lymphadenectomy. J Am Coll Surg 2000;191:114-9.

27. Japanese Gastric Cancer Association. Japanese gastric cancer treatment guidelines 2010 (ver. 3). Gastric Cancer 2011;14:113-23.

28. Cuschieri A, Fayers P, Fielding J, et al. Postoperative morbidity and mortality after D1 and D2 resections for gastric cancer: preliminary results of the MRC randomized controlled surgical trial. The Surgical Cooperative Group. Lancet 1996;347:995-9.

29. Japanese Gastric Cancer Association. Japanese classification of gastric carcinoma--2nd English edition-response assessment of chemotherapy and radiotherapy for gastric carcinoma: clinical criteria. Gastric Cancer 2001;4:1-8.

30. Yao ZD, Yang H, Cui M, et al. Application of transorally inserted anvil (OrVil(TM)) in laparoscopic-assisted radical resection for Siewert type II adenocarcinoma of the esophagogastric junction. Zhonghua Wei Chang Wai Ke Za Zhi 2013;16:345-9.

31. Yamashita H, Seto Y, Sano T, et al. Results of a nationwide retrospective study of lymphadenectomy for esophagogastric junction carcinoma. Gastric Cancer 2017;20:69-83.

32. Wang J, Wu P, Wang Z, et al. Metastatic patterns and surgical methods for lymph nodes No. 5 and No. 6 in proximal gastric cancer. Chin J Cancer Res 2019;31:171-7.

33. Ushimaru Y, Fujiwara Y, Shishido Y, et al. Clinical Outcomes of Gastric Cancer Patients Who Underwent Proximal or Total Gastrectomy: A Propensity ScoreMatched Analysis. World J Surg 2018;42:1477-84.

34. Jung DH, Lee Y, Kim DW, et al. Laparoscopic proximal gastrectomy with double tract reconstruction is superior to laparoscopic total gastrectomy for proximal early gastric cancer. Surg Endosc 2017;31:3961-9.

35. Zhang $\mathrm{C}, \mathrm{Hu} \mathrm{X}$. Function-preserving gastrectomy for early gastric cancer based on Japanese researches. Zhonghua Wei Chang Wai Ke Za Zhi 2018;21:148-53.

36. Takiguchi N, Takahashi M, Ikeda M, et al. Long-term quality-of-life comparison of total gastrectomy and proximal gastrectomy by Postgastrectomy Syndrome Assessment Scale (PGSAS-45): a nation-wide multiinstitutional study. Gastric Cancer 2015;18:407-16.

37. Asaoka R, Irino T, Makuuchi R, et al. Changes in body weight, skeletal muscle and adipose tissue after gastrectomy: a comparison between proximal gastrectomy and total gastrectomy. ANZ J Surg 2019;89:79-83.

38. Sugiyama M, Oki E, Ando K, et al. Laparoscopic Proximal Gastrectomy Maintains Body Weight and Skeletal Muscle Better Than Total Gastrectomy. World J Surg 2018;42:3270-6.

39. Nakamura H, Murakami Y, Morifuji M, et al. Analysis of fat digestive and absorptive function after subtotal gastrectomy by a 13C-labeled mixed triglyceride breath test. Digestion 2009;80:98-103.

40. Shibata C, Ueno T, Kakyou M, et al. Results of reconstruction with jejunal pouch after gastrectomy: correlation with gastrointestinal motor activity. Dig Surg 2009;26:177-86.

41. Katai H, Mizusawa J, Katayama H, et al. Single-arm confirmatory trial of laparoscopy-assisted total or proximal gastrectomy with nodal dissection for clinical stage I gastric cancer: Japan Clinical Oncology Group study JCOG1401. Gastric Cancer 2019;22:999-1008.

42. Song $\mathrm{W}$, Liu Y, Ye J, et al. Proximal gastric cancer: lymph node metastatic patterns according to different T stages dictate surgical approach. Chin Med J (Engl) 2014;127:4049-54.

43. Wang W, Zheng C, Fang C, et al. Time trends of clinicopathologic features and surgical treatment for gastric cancer: Results from 2 high-volume institutions in southern China. Surgery 2015;158:1590-7.

44. Nomura E, Isozaki H, Fujii K, et al. Postoperative evaluation of function-preserving gastrectomy for early gastric cancer. Hepatogastroenterology 2003;50:2246-50.

45. Kudou K, Saeki H, Nakashima Y, et al. Postoperative development of sarcopenia is a strong predictor of a poor prognosis in patients with adenocarcinoma of the esophagogastric junction and upper gastric cancer. Am J Surg 2019;217:757-63.

46. Jung DH, Ahn SH, Park DJ, et al. Proximal Gastrectomy for Gastric Cancer. J Gastric Cancer 2015;15:77-86.

(English Language Editor: J. Jones)

Cite this article as: Ma F, Guo D, Zhang B, Zhang Y, Peng L, Ma Q, Ji S, Chai J, Hua Y, Chen X, Wang H, Xu S, Luo S. Short and long-term outcomes after proximal gastrectomy with double tract reconstruction for Siewert type III adenocarcinoma of the esophagogastric junction: a propensity score matching study from a 10-year experience in a high-volume hospital. J Gastrointest Oncol 2020;11(6):1261-1273. doi: 10.21037/jgo-20-475 